\title{
CESÁREA POR SOLICITUD MATERNA (CPSM)
}

a cesárea es el procedimiento quirúrgico más frecuentemente realizado en mujeres, y su objetivo es disminuir la morbimortalidad materna-perinatal. Históricamente, las indicaciones han sido diversas. En la antigua Roma, la Lex Caesarea contemplaba que a una mujer que muriese en el parto se le debía realizar esta cirugía con el fin de salvar la vida del feto; antes del siglo XVI, y por influencia religiosa, era obligatorio realizarla en toda mujer que falleciera antes del parto para enterrar los cuerpos de la madre y el bebé por separado (1). Es solo hasta el siglo XVI que se conoce dato de la primera mujer que sobrevivió a una cesárea y, desde entonces, las indicaciones habían estado reservadas a casos en que se presumía que el parto vaginal era arriesgado para la madre o el feto, con un aumento en su frecuencia asociado a la seguridad de los procedimientos quirúrgicos, las técnicas anestésicas y los cambios culturales tanto de los médicos como de las mujeres (2). Recientemente, se ha incorporado el concepto de cesárea por solicitud materna (CPSM), la cual se presume debe ser debidamente informada respecto a los beneficios y riesgos materno-fetales a corto y a largo plazo.

En el 2015, la Organización Mundial de la Salud (OMS) consideró que la tasa ideal de cesárea debía ser menor al $15 \%$, y que tasas superiores al $10 \%$ no se asociaban con reducción de la mortalidad materna o de los recién nacidos (3). Sin embargo, a pesar de esta recomendación, en el mundo la tasa de cesáreas ha venido aumentando constantemente, siendo en Estados Unidos, en 1989, alrededor de 23\%, y en 2011 de 33 \% (4). En Colombia, en 1998, la proporción de cesáreas fue 24,9\% y en 2014 de 46,6\% (5), lo que muestra un aumento mayor que en Estados Unidos. Aunque la tasa de mortalidad perinatal en Colombia por cada mil embarazos de más de 7 meses disminuyó de 24 a 14 por mil en el periodo 2000-2010, y la tasa de mortalidad neonatal de 7,5 a 5,6 por mil nacidos vivos entre 2005-2012 (5), estas reducciones no se relacionan con el aumento epidémico en la tasa de cesáreas.

No hay estadísticas confiables respecto al impacto de la CPSM en la tasa general de cesárea en nuestro medio, pero se presume que ha aumentado, al igual que en otros países. En Estados Unidos se estima que representa entre el 4-18\% de todas las cesáreas (6), en Escocia el 7,7\% (7) y en el norte de Australia el 26,8 \% (8), con diferentes grados de aceptación por parte del personal médico. Entre 2001 y 2002 el grado de aceptación por parte de obstetras norteamericanos fue de 46\% (9), mientras en el 2006, de 1031 ginecólogos de la ACOG, $20 \%$ reportaron que solicitarían una cesárea por requisito para su esposa y $53 \%$ reconocieron haber realizado cesáreas por la misma causa una o dos veces al mes (10). En el mismo año, en España, de 1222 ginecólogos adscritos a la SEGO, 57,8\% no aceptarían realizar una cesárea por solicitud materna en caso de gestante primigestante con feto en presentación cefálica, mientras 24,8 \% sí lo harían, el restante 17,4 \% no tomó ninguna posición (11). No se conoce el grado de aceptación de CPSM por parte de los ginecólogos en Colombia, pero se presume que es alto, lo cual puede reflejar en parte el aumento dramático de este procedimiento en los últimos años, más acentuado en algunas regiones del país, como en los departamentos de la región Caribe (5). 
No hay en la actualidad estudios de buena calidad que determinen los riesgos y beneficios de la CPSM, los pocos que se encuentran son retrospectivos y se limitan a presentar los resultados a corto plazo. En una revisión sistemática de estudios de cohorte y de casos y controles realizada por el Instituto Nacional de Salud de Estados Unidos (12) se encontró que, para la madre, solo dos resultados a corto plazo -hemorragia y estancia hospitalaria materna- alcanzaron un nivel de evidencia moderada, con menor frecuencia de hemorragia posparto en caso de cesárea planeada, comparada con cesárea no planeada y parto vaginal planeado; por el contrario, la estancia fue mayor en casos de cesárea comparados con parto vaginal, aunque los resultados de la cesárea incluyen tanto la planeada como la no planeada. Con una débil calidad de evidencia se encontró menor frecuencia de infecciones, complicaciones anestésicas, placenta previa y abandono de la lactancia en gestantes con parto vaginal planeado. En el caso de infecciones, estas fueron menores en la cesárea planeada en comparación con la no planeada; respecto a complicaciones anestésicas, la mayoría se asocia a anestesia general, más frecuentemente usada en cesárea de emergencia, mientras en caso de cesárea planeada generalmente se opta por anestesia regional, la cual tiene menos riesgo de complicaciones que la general. Respecto a placenta previa, cada vez es más consistente la evidencia del aumento de riesgo con el antecedente de cesárea y el número de cesáreas previas con el concomitante aumento de riesgo de acretismo placentario (13) y sus complicaciones, como necesidad de histerectomía, transfusión de productos sanguíneos, ingreso a unidad de cuidados intensivos y complicaciones trombóticas.

En la revisión sistemática mencionada (12), se encontró débil calidad de evidencia a favor de CPSM para incontinencia urinaria (IU), fecal (IF) y trauma obstétrico materno; no obstante, no es claro si el aumento de frecuencia de IU e IF en caso de parto vaginal planeado permanece en el tiempo y, aparentemente, no hay diferencias cuando la mujer alcanza los 50 años; adicionalmente, esta incontinencia se encuentra más relacionada con el número de embarazos y la edad materna que con la vía de finalización de la gestación.

En relación con los resultados neonatales, se encontró moderada calidad de evidencia a favor del parto planeado en morbilidad respiratoria como consecuencia de mayor frecuencia de taquipnea transitoria del recién nacido y síndrome de distrés respiratorio leve en caso de cesárea planeada, con muy baja frecuencia de falla respiratoria severa o hipertensión pulmonar. Con débil calidad de evidencia se encontró menor estancia hospitalaria del neonato en caso de parto planeado, por el contrario, se encontró menor frecuencia de mortalidad fetal, hemorragia intracraneal y fractura de clavícula en caso de cesárea planeada; no obstante, la mortalidad fetal asociada a parto se presentó por encima de las 41 semanas. En la actualidad, la mayoría de instituciones promueven la finalización de la gestación antes de la semana 41, con lo cual esta posible diferencia tiende a minimizarse.

En nuestro medio, el primer estudio que compara el impacto perinatal de la cesárea por requisito de la embarazada se publica en este volumen, por el grupo de Sarmiento-Rodríguez en una institución hospitalaria universitaria privada. Se trata de un estudio de cohorte prospectivo, realizado en 931 gestantes entre 18 y 45 años, de bajo riesgo, con embarazo a término (edad gestacional mayor de 37 semanas) durante el periodo comprendido entre junio de 2008 y abril de 2012, en el cual las gestantes fueron invitadas a participar y a firmar el consentimiento informado antes de la semana 36 durante su consulta de control prenatal. De las 931 gestantes, a 214 (22,99\%) se les realizó cesárea por solicitud materna (CPSM), 341 (36,63\%) iniciaron trabajo de parto espontáneo (TPVE) y 376 $(40,38 \%)$ iniciaron trabajo de parto inducido (TPI) por razones médicas, obstétricas o evidencia de estado fetal no satisfactorio. El resultado principal materno fue una variable compuesta denominada resultado materno, que incluyó alguna complicación como necesidad de transfusión, histerectomía, cuidados intensivos, trauma obstétrico e infección puerpe- 
ral. El resultado primario neonatal es otra variable compuesta que incluye Apgar a los 5 minutos menor de 7, bajo peso, cefalohematoma, ictericia, hipoglicemia, hipokalemia, sepsis neonatal, taquipnea transitoria del recién nacido, enfermedad de membrana hialina, enterocolitis necrotizante, neumonía, asfixia, aspiración de meconio, eventos potencialmente peligrosos, malformaciones, necesidad de intubación y muerte neonatal.

Los autores encuentran menor riesgo de resultados maternos adversos en el grupo de CPSM respecto al grupo de TPVE (OR = 0,21; IC 95\%: 0,05-0,97), sin diferencias entre TPVE y TPI (OR = 0,93; IC 95\%: $0,42-2,06)$ y en los resultados primarios neonatales encuentran también menor riesgo en el grupo de CPSM respecto a grupo de TPVE $(\mathrm{OR}=0,59$; IC 95\%: 0,36-0,93), sin diferencias entre TPVE y TPI $(\mathrm{OR}=0,84$; IC 95\%: 0,59-1,21), por lo cual concluyen que en embarazadas de bajo riego que entran en un protocolo estandarizado la CPSM tiene más baja tasa de resultados maternos y perinatales adversos, y plantean la necesidad de futuros estudios para determinar la necesidad de la seguridad a largo plazo.

Los resultados de este estudio podrían alentar a la comunidad médica a estimular la CPSM, sin embargo, se deben interpretar con cautela por varias razones:

1. Los autores no reportan el resultado de todas las pacientes invitadas a participar en el estudio; como ellos mencionan, la invitación se realizó antes de la semana 36, sin embargo, los resultados solo incluyen las gestantes que alcanzaron la semana 39 (sesgo de desgaste) y se desconoce la proporción de gestantes no incluidas en los resultados, como también el resultado de las mismas por lo cual no es posible determinar la dirección en la cual se afecta el estimador obtenido (OR). Si se tienen en cuenta resultados de estudios previos que muestran mayor número de complicaciones en las pacientes llevadas a cesárea electiva frente a TPVE, la no inclusión de estas gestantes favorece el encontrar resultados a favor de la CPSM.
2. Los resultados maternos y fetales se presentan como una variable compuesta, lo cual tiene beneficios al aumentar el poder del estudio; no obstante, la combinación de variables oculta lo que sucede con cada una de ellas y no todos los resultados tienen el mismo impacto clínico (14). En los resultados maternos se encuentra que en el grupo de TPVE hubo menor necesidad de transfusión que en el de CPSM $(0,3 \%$ vs. $0,5 \%$ ) y mayor frecuencia de trauma obstétrico materno $(2,1 \%$ vs. $0 \%)$, pero no se menciona qué se consideró trauma obstétrico, como tampoco las causas que llevaron a la necesidad de transfusión, complicación esta que puede constituir un criterio de morbilidad materna extremadamente grave. En cuanto a los resultados neonatales, la complicación más frecuente fue la ictericia, correspondiendo al 78,84\% (149 de 189) del total de complicaciones del neonato, entidad esta que es poco plausible que se relacione con la vía de nacimiento, a diferencia del Apgar bajo a los 5 minutos, muerte neonatal, aspiración de meconio, transición inadecuada, taquipnea transitoria del recién nacido y necesidad de intubación, que sí guardan una relación con la vía de nacimiento, pero fueron poco frecuentes, siendo la taquipnea transitoria la de mayor incidencia, especialmente en el grupo de cesárea, seguida por la transición inadecuada la cual fue más frecuente en el grupo de TPVE.

3. En el estudio también se compara la cohorte de gestantes llevadas a CPSM con las de TPI, con más frecuencia de complicaciones en estas últimas; sin embargo, es de anotar que los TPI fueron por indicaciones maternas u obstétricas, o por sospecha de estado fetal no satisfactorio, lo cual constituye un sesgo de selección que tiende a que se encuentren peores resultados en este grupo respecto a las cohortes de TPVE y CPSM.

4. El 84,6\% de la población incluida en el estudio tiene como asegurador una entidad de medicina prepagada, a diferencia de la mayor parte de la población de gestantes colombianas que pertenecen al régimen subsidiado o al régimen contributivo, por lo cual los resultados no son generalizables. 
En el ejercicio de la medicina se deben tener en cuenta principios éticos como el de la beneficencia, que consiste en ofrecer a los pacientes prácticas que aumenten los beneficios y disminuyan los riesgos. Con respecto a la vía de finalización del embarazo la evidencia es consistente en un menor riesgo y mayor beneficio tanto materno como fetal en el parto con respecto a la cesárea; no obstante, para el caso de la cesárea electiva y CPSM, después de la semana 39, aparentemente esta diferencia entre los beneficios y riesgos en relación con el TPVE parece equilibrarse, sin que a la fecha se tenga evidencia de buena calidad que permita al personal médico recomendar la cesárea en una forma contundente. Por otra parte, el principio de la autonomía, que trata de garantizar a los pacientes decidir respecto a la aceptación o no de las intervenciones ofrecidas por el personal de salud, permite la solicitud de cesárea por parte de la gestante, la cual debe ser respetada, pero esta decisión debe ser consciente, en una paciente debidamente informada acerca de los beneficios y riesgos demostrados; empero, en un estudio (15) se encontró que entre los principales determinantes de una gestante para decidir la vía de finalización del embarazo se encuentra el papel del médico tratante, siendo más relevante cuando desea parto vaginal y la decisión se transforma finalmente en petición de cesárea.

En conclusión, la CPSM es una intervención que viene en aumento, respecto a la cual no hay evidencia de alta calidad que demuestre que tenga mayores beneficios y menores riesgos que el parto vaginal espontáneo a corto plazo, y no hay estudios que evalúen la frecuencia de complicaciones a largo plazo, como placenta previa, acretismo placentario y embarazo ectópico en la cicatriz de la cesárea previa. La decisión de la gestante de solicitar una cesárea debe ser mediada por previa información de los beneficios y riesgos de este procedimiento a corto y a largo plazo, lo cual generalmente no se logra en una sola consulta, por el contrario, es un proceso continuo que se debe realizar durante todo el control prenatal. En esta decisión inevitablemente juega un papel importante el médico tratante, quien puede influenciar a la ma- dre de acuerdo con sus conocimientos, creencias y conveniencia.

\section{Javier E. Fonseca-Pérez MD, MSc}

Editor invitado

Profesor Asistente

Departamento de Obstetricia y Ginecología

Universidad del Valle

\section{REFERENCIAS}

1. Sánchez F. La operación cesárea en Colombia (recuento histórico). Rev Colomb Obstet Ginecol. 1971;XXII:101-10.

2. Vázquez Parra, JC. Abuso de la operación cesárea y el principio de beneficencia. Revista Latinoamericana de Bioética. 2016;16:60-71.

3. Gibbons L, Belizán JM, Lauer JA, Betran AP, Merialdi $\mathrm{M}$, Althabe F. The global numbers and costs of additionally needed and unnecessary caesarean sections performed per year: Overuse as a barrier to universal coverage. Geneva: World Health Organization; 2010. Report No. 30.

4. Hamilton BE, Hoyert DL, Martin JA, Strobino DM, Guyer B. Annual summary of vital statistics: 20102011. Pediatrics. 2013;131:548-58.

5. Rubio-Romero JA, Fonseca-Pérez JE, Molina-Giraldo S, Buitrago-Leal M, Zuleta-Tobón JJ, Ángel-Muller E et al. Racionalización del uso de la cesárea en Colombia. Consenso de la Federación Colombiana de Obstetricia y Ginecología (Fecolsog) y la Federación Colombiana de Perinatología (Fecopen). Rev Colomb Obstet Ginecol. 2014;65:47-74.

6. National Institutes of Health State-of-the-Science Conference Statement Cesarean Delivery on Maternal. Editorial. Obstet Gynecol. 2006;107:1386-97.

7. Wilkinson C, Mc Ilwaine G, Boulton-Jones C, Cole S. Is a rising caesarean section rate inevetable? $\mathrm{Br} \mathrm{J}$ Obstet Gynaecol. 1998;105:45-52.

8. Quinlivan JA, Petersen RW, Nichols CN. Patient preference the leading indication for elective caesarean section in public patients-results of a 2 -year prospective audit in a teaching hospital. Aust N Z J Obstet Gynaecol. 1999;39:207-14. 
9. Gabbe GS, Holzman GB. Obstetrisian's choce of delivery. Lancet. 2001;357:722.

10. Bettes BA, Coleman VH, Zinberg S, Spong CY, Portnoy B, DeVoto E et al. Cesarean delivery on maternal request: obstetrician-gynecologists' knowledge, perception, and practice patterns. Obstet Gynecol 2007;109:57-66.

11. De la Fuente P, De la Fuente L. Cesárea a petición de la embarazada. Encuesta de opinión de la Sociedad Española de Obstetricia y Ginecología. Prog Obstet Ginecol. 2007;550:420-8.

12. National Institutes of Health State-of-the-science Conference Stament. Cesarean Delivery on Maternal Request. Obstet Gynecol. 2006;107:1386-97.
13. Silver RM, Landon MB, Rouse DJ, Leveno KJ, Spong $\mathrm{C}$, Thom EA et al. Maternal morbidity associated with multiple repeat cesarean deliveries. Obstet Gynecol. 2006;107:1226-32.

14. Montori VM, Permanyer-Miralda G, Ferreira-González I, Busse JW, Pacheco-Huergo V, Briant D, et al. Validity of composite end points in clinical trials. BMJ. 2005;330:594-6.

15.Shams-Ghahfarokhi Z, Khalajabadi-Farahani F. Intention for cesarean section versus vaginal delivery among pregnant women in Isfahan: Correlates and determinants. J Reprod Infertil. 2016;17230-9. 